\section{SYPHILIS IN THE DIAGNOSIS AND PROGNOSIS OF CANCER}

BY

J. I. MUNRO BLACK, M.S., F.R.C.S.

Junior Assistant Pathologist, late Assistant Radium Officer, Junior Surgical Registrar, Royal Victoria Infirmary, Newcastle-upon-Tyne

The association of syphilis and cancer has long been appreciated as one of some closeness, and much work has been done on the subject and interesting results obtained from its study. Most of this has centred upon syphilis as an aetiological factor, and is, of course, of great importance in the appreciation of pre-cancerous conditions. The practical application, however, of this work must lie with the authorities concerned in the control of venereal disease, and with those who have to treat syphilis. From the point of view of the surgeon dealing with a cancerous patient it may be of great philosophical interest to know that a history of syphilis is present, and of course if the disease is still active the patient should be handed over to the proper person for full anti-syphilitic treatment later. This paper is concerned, however, not with pre-existing syphilis and cancer, but with the danger of coexisting syphilis giving rise to difficulties in the diagnosis of cancer, and perhaps modifying the prognosis by causing delay in treatment.

The universality of syphilis, especially in the days not long past, and the multiplicity of the lesions in its later stages, has given it the place of honour as the producer of any unusual condition. In consequence one finds a tendency to think of syphilis when confrcented with any curious lump or ulcer. For the older generation of surgeons it may have been common to find the lesions of tertiary syphilis, but to a younger generation they are of comparative rarity, and it is the fostering of this newer outlook, especially with regard to the diagnosis of cancer, that I wish to encourage. If a gummatous lesion is not diagnosed it may be very gratifying for someone more experienced to point out the obvious nature of the lesion and the ease of its cure, but no great harm will be done by any delay incurred.

\section{Two Illustrative Cases}

An outstanding example comes to mind in the case of a young man, aged 21, who was seen in October, 1934, with a swelling in the right temporal region of four months' duration.

The swelling had appeared originally with a little pain a week after a local injury, since when it had steadily increased in size with slight variation. The swelling was diffuse, a little tender, and rather "boggy." It was incised, but no pus was obtained, and a radiograph did not show any changes in the underlying bone or other abnormality. The patient returned two months later with the mass increased in size, and it was treated with interstitial radium. This had no effect, and a month later the mass was excised and was shown by histological examination to be a gumma. With this knowledge a Wassermann reaction was carried out and proved to be positive.

Indeed a sorry tale, all of which could have been obviated by a biopsy at the very beginning, but the great fact remains that the man is alive and has now had a full course of anti-syphilitic treatment.

A man, aged 26, was sent to hospital with a diagnosis of carcinoma of the tongue, confirmed by biopsy, and with a negative Wassermann. The lesion, however, did not look neoplasțic, and a further biopsy was performed, which was reported as probably gummatous in spite of the negative Wassermann. This reaction was repeated and proved positive, and the patient was given potassium iodide. Rapid improvement resulted, and was followed by a full course of proper anti-syphilitic treatment. In this case the true diagnosis was arrived at first by experienced clinical observation, but ultimately by the opinion of a good histologist.

On the other hand, if a cancerous lesion is treated as syphilitic, then the real diagnosis will probably only be evidenced by the appearance of metastases, by which time the outlook is very gloomy. Many patients are unfortunate enough to suffer from cancer and have serological evidence of syphilis. In their cases either a false diagnosis is made or doubt left owing to the positive Wassermann reaction, and valuable time may be lost while potassium iodide is given, or even a full course of anti-syphilitic treatment.

\section{Diagnostic Difficulties in Oral and Pharyngeal Cancer}

It has been found that the difficulties of diagnosis seem particularly great in oral and pharyngeal cancer or in associated neck glands, and it is with special reference to such cases that I write, although I believe my remarks have a very general import. In the first place, cases are seen in which the diagnosis of the lesion has been uncertain, and the practitioner has fallen back on syphilis or cancer as possible causal agents. It is very easy to take blood for a Wassermann reaction, and if this should happen to be positive-and there is a greater possibility of such being the case in a cancer patientthen potassium iodide will probably be given. Now it is well known that malignant lesions, especially those of the mouth, do show evident-though, of course, only temporary-improvement under potassium iodide ; so that it is possible that the combined influence of a positive Wassermann and improvement under potassium iodide might lead to a full course of anti-syphilitic treatment being started. This is perhaps a good programme for the treatment of a gumma, granting that to a surgeon familiar with the manifestations of tertiary syphilis the lesion would probably have been obvious in the first place. Owing to more efficient control and early treatment of syphilis, such lesions and such surgeons are becoming rare. If, however, the lesion be not syphilitic, but cancerous, a tragedy will be well on its way to occurring. Such a danger is very real, and is brought forcibly to notice when one sees how closely a cancer can at times mimic a gumma.

A case in point was that of a male, aged 54 , who was seen in June, 1936, with an ulcer in the middle line of the middle third of the dorsum of his tongue, about $3 \mathrm{~cm}$. in diameter. The base was formed by a yellowish, sloughing mass, but the edge was slightly raised and hard. There was no fixation of the tongue, and no enlarged neck glands were present. The surrounding tongue and palate were leukoplakic. The ulcer had begun as a little lump six weeks previously, and had not caused any pain. A tentative diagnosis of gumma had been made, and many who saw the case agreed that this was correct. Owing, however, to the suspicious nature of the edge a biopsy was asked for, and at the same time a Wassermann reaction was carried out-and incidentally reported as positive. The histological examination revealed a non-keratinizing squamous carcinoma. Radium treatment was started, and later a block dissection of the neck was performed.

In such a rapidly growing tumour the outlook could not be good, and the patient has now a recurrence in his neck, but any delay which would surely have occurred in the absence of a biopsy would have made things very much worse. 


\section{The Question of Biopsy}

It is better to be ever thinking of cancer at the outset, and to make the diagnosis by biopsy if there is doubt. Should there then be a suspicion of syphilis, the question of cancer having been ruled out, let a Wassermann reaction be confirmatory only, and hand the patient over to the proper person for full treatment. An objection to this line of investigation is the danger of the biopsy enhancing the possibility of metastasis. Personally I have not seen or heard of any damage resulting, but in view of other better qualified opinions I must acknowledge the possibility. Perhaps the diathermy needle helps to minimize this risk, and in dealing with the more malignant anaplastic carcinomata and sarcomata, in which the danger is supposedly much greater, a dose of $x$ rays might be given immediately before or after the biopsy. The extreme importance of early accurate diagnosis is, however, so great that some risk is justifiable. We are left with the possibility in the highly malignant cases of using irradiation as a means of diagnosis as well as treatment should a biopsy really be contraindicated. A rapid resolution will suggest the continuance to a full course of treatment: no response will demand a diagnosis.

A second type of case occurs in which the lesion is or should be recognizable as a cancer, but the patient is found to have a positive Wassermann reaction. In many clinics such an investigation is carried out as a routine on all possible cancer cases. In the past this was of importance for completeness of records or for statistics, which I venture to suggest have now proved the aetiological relationship of the two conditions. When done in a cancer clinic or under the auspices of one who sees many cancer cases, and who is accordingly ever on the look-out for neoplasms, little harm can occur, but if done under less favourable circumstances there may be a tendency to treat the syphilis first and see what happens to the suspect lesion. In such an event $I$ am of the opinion that really valuable time is lost. The cancer must be treated first: after all, it does not take long, and then the patient can be handed over for full and proper antisyphilitic treatment. Remembering that there is real danger of a coincidental positive Wassermann reaction throwing doubt upon the diagnosis of a cancerous lesion, one wonders whether anything is gained by its routine performance in this type of case. This is really of vital importance, because we are dependent for better results upon earlier diagnosis, and this earlier diagnosis is going to be made, not by cancer experts, but by the general practitioners, who must at all costs play for safety and think of cancer first. It is not suggested that the general practitioners should themselves perform a biopsy upon all doubtful lesions, because a surgeon of greater experience may be confident of the diagnosis on clinical grounds. But if after consultation some doubt remains, then biopsy must be urged, and should be performed by one who is in close co-operation with a pathologist.

A final possibility is that in leaving the syphilis untreated during the treatment of the cancer the prognosis may be worsened, but I do not know of any evidence to this effect, and do not see any real reason why antisyphilitic treatment should not be started immediately in any case. That, however, is not a question for the surgeon or radiologist who is dealing with the cancer, but for the venereologists, and I maintain that the canser has the prior claim to treatment.

\section{Clinical Memoranda}

\section{The Aetiology of Femoral Hernia}

When operating recently on a man of 59 for strangulated femoral hernia I found a condition that interested me in the light of the theories of origin.

\section{CASE REPORT}

This particular hernia had existed for thirteen or fourteen years ; its development had been gradual, and up to the time of the patient's present illness it had been reducible. The onset of pain was sudden, with vomiting; I saw him about three hours later. Efforts at reduction failed, and operation was undertaken. Incision over the tumour showed an engorged purple mass, having a superficial resemblance to bowel; it proved, however, to consist of lobules of fat. It was followed up to a narrow neck, constricted as usual by the sharp edge of Gimbernat's ligament. After prolonged dissection the sac was discovered and opened, inside the strangulated mass, and was quite empty. The tumour consisted of extraperitoneal fat on the outer surface of an empty hernial sac. (It is of interest that, when exposing the external abdominal ring in the course of operation, a lobule of extraperitoneal fat projected here also, and was sliding freely up and down the inguinal canal.) The sac was tied off in the femoral canal, the stump drawn up above Poupart's ligament, and stitched to the external limb of the conjoined tendon; the canal was then closed by suture through conjoined tendon, Cooper's ligament, and external oblique, at the same time bringing the medial wall of the internal abdominal ring beneath the cord into apposition with the upper and internal surface of the external oblique, as for Bassini's closure.

\section{COMMENTARY}

I record this case as it seems to support J. Philip Buckley's (1934) "acquired saccular" theory of origin, by which, as he supposes, extraperitoneal fat protrudes through the femoral ring, expands in the loose tissues of the thigh, and draws down further lobules and eventually a small pouch of attached peritoneum. A time comes when the expanded fatty mass is unable to return through the narrow neck. It would seem obvious that in my patient at any rate this had been the course of events. R. Hamilton Russell (1923) asserts that femoral sacs are invariably congenital, acquiring contents at varying periods of life, and that removal of the sac is therefore the only treatment necessary. In cases such as mine, where the sac appeared to be an unimportant adjunct to a mass of extraperitoneal fat, it seems doubtful if this treatment would be adequate. The theory also fails to explain how recurrent femoral hernias occur after operation and removal of the sac. Leslie W. Tasche (1932), on the other hand, finds that these sacs are never congenital. There is at least no record of their discovery in a foetus or a newborn infant. He also holds that traction rather than pressure is the primary cause of the hernia, rejecting Murray's theory of abnormal gubernacular pull, for which there seems very little evidence, and believing that the primary escape is of a fat lobule, a sac and its possible contents being drawn down subsequently.

When one comes to consider treatment, it seems at least doubtful whether removal of the sac, without any further precautions, would in the case here recorded have been an adequate safeguard against recurrence or redevelopment.

Bourne, Lincs. $\quad$ W. B. R. MonTEITH, F.R.C.S.Ed.

Buckley, J. Philip (1924). Brit. J. Surg. 12, 60

Russell, R. Hamilton (1923). Ibid., 11 , 148 .

Tasche, Leslie W. (1932). Arch. Surg., Chicago, 25, 749. 